\title{
Mycobacterium Wolinskyi: A New Non-Tuberculous Mycobacterium Associated with Cardiovascular Infections?
}

\author{
Marta Hernández-Meneses · Julian González-Martin · Daiana Agüero • \\ Jose M. Tolosana - Elena Sandoval · Carles Falces · Rodolfo San Antonio · \\ Bárbara Vidal · Asunción Moreno · Juan Ambrosioni (1) · \\ Jose M. Miró · Hospital Clínic of Barcelona Infectious Endocarditis Team
}

Received: December 29, 2020 / Accepted: February 10, 2021 / Published online: March 15, 2021

(C) The Author(s) 2021

\section{ABSTRACT}

Mycobacterium wolinskyi is a rapid-growth nontuberculous mycobacterium. Twenty-one cases of M. wolinskyi infection have been described so far, more than half as cardiovascular or postoperative cardiothoracic infections. We report the case of a patient with a cardiovascular implantable electronic device infected by $M$.

Members of the Hospital Clinic Endocarditis Study Group are listed in "Acknowledgements" section.

Supplementary Information The online version contains supplementary material available at https:// doi.org/10.1007/s40121-021-00416-8.

M. Hernández-Meneses · D. Agüero · A. Moreno · J. Ambrosioni $(\bowtie) \cdot J$. M. Miró $(\bowtie)$ Infectious Diseases Department, Hospital ClinicIDIBAPS, University of Barcelona, Barcelona, Spain e-mail: jambrosioni@intramed.net

J. M. Miró

e-mail: jmmiro@ub.edu

J. González-Martin

Microbiology Dept-CDB, Hospital Clinic-ISGlobal-

University of Barcelona, Barcelona, Spain

J. M. Tolosana - C. Falces · R. San Antonio - B. Vidal Cardiology Service, Hospital Clinic-IDIBAPS,

University of Barcelona, Barcelona, Spain

E. Sandoval

Cardiovascular Surgery Service Hospital Clinic-

IDIBAPS, University of Barcelona, Barcelona, Spain wolinskyi, successfully treated with device removal and antimicrobials.

Keywords: Cardiac implantable electronic device infections; Cardiovascular infections; Mycobacterium wolinskyi; Non-tuberculous mycobacteria; Sternal osteomyelitis

\section{Key Summary Points}

Mycobacterium wolinskyi may be an emerging non-tuberculous mycobacterium (NTM) specifically associated with cardiovascular infections

Mycobacterium wolinskyi has been shown to be capable of producing a biofilm and causing outbreaks such as nosocomial infection

The Mycobacterium wolinskyi treatment strategy is poorly understood. The combination therapy scheme could include quinolones, tetracyclines, linezolid, carbapenems and cotrimoxazole

NTM infections are uncommon but should be considered when cultures remained negative for common pathogens, and molecular biology should be used to increase the diagnostic yield 


\section{DIGITAL FEATURES}

This article is published with digital features, including a summary slide, to facilitate understanding of the article. To view digital features for this article go to https://doi.org/10.6084/ m9.figshare.13806977.

\section{INTRODUCTION}

Mycobacterium wolinskyi is a rapid-growing nontuberculous mycobacteria (NTM), belonging to the Mycobacterium smegmatis group [1, 2], which can be isolated from various environmental settings, such as soil and water. M. wolinskyi was first identified by Brown et al. in 1999 using 16S rRNA gene sequencing [1]. The ability of NTMs to infect immunocompromised patients has long been described, but their prevalence has increased over recent years in both immunocompromised and immunocompetent patients, mostly in healthcare settings [2].

Most cases of NTM infections in cardiovascular patients published in the literature are related to the recent outbreak of Mycobacterium chimaera and contamination of water heater and cooler tanks [3-5]. However, the increasing prevalence of cardiovascular infections due to NTMs is not limited to this microorganism. Since M. wolinskyi was described in 1999, only a few cases have been published in the literature, and more than half were cardiovascular or postoperative infections [9-12]. Here, we report a case of an early cardiac implantable electronic device (CIED) infection due to $M$. wolinskyi that required complete device removal and combined antimicrobials for successful treatment. A review of the literature is also presented.

\section{METHODS}

We present our clinical case description and microbiologic findings and a review of the literature in MEDLINE (source PubMed) from 1999 to April 2020 under the criteria of infection, specifically NTM and $M$. wolinskyi infection, including papers in all published languages. The patient provided informed consent for publication. Of all the identified cases, only those with cardiovascular involvement were retained, and their characteristics, management and evolution, including our case, were analyzed. The patient has provided informed consent for the publication of this case report.

\section{CASE REPORT}

A 63-year-old woman was referred to our center with a clinical history of 10 days of general weakness, swelling, tenderness and purulence at the site of a cardiac resynchronization therapy (CRT) device implanted one month earlier. She denied experiencing chills or fever. Past medical history included hypertension, atrial fibrillation, dilated non-ischemic cardiomyopathy (ejection fraction 25\%) and recent cardio-embolic stroke with complete neurologic recovery 6 weeks earlier. Upon hospital admission, her physical examination revealed a body temperature of $37.2{ }^{\circ} \mathrm{C}$, blood pressure of $160 / 80 \mathrm{mmHg}$, pulse of 100 beats/min and normal heart sounds, but no other abnormalities except those described at the pocket site of the CRT on the right chest wall. Significant laboratory test results included a white blood cell count of $6.3 \times 109 / \mu \mathrm{l}(4-11 \times 109 / \mu \mathrm{l})$, C-reactive protein $12.7 \mathrm{mg} / \mathrm{l}(<10 \mathrm{mg} / \mathrm{l})$ and normal renal and liver function tests. Four sets of aerobic and anaerobic blood cultures were collected at that time, all of them negative. Swabs and aspiration drainage from the pocket site were also collected for cultures and $16 \mathrm{~S}$ ribosomal RNA gene (rRNA) PCR/sequencing. Chest $\mathrm{x}$-ray was normal, and transthoracic and transesophageal echocardiography showed no vegetations or thrombus on either the valve or the leads. The CIED was removed 5 days after admission, and all the samples (pocket swab, CRT pocket and leads) were remitted to microbiology for standard cultures, and all the samples were processed for rRNA gene sequencing. The patient progressed with her own cardiac rhythm, not requiring further temporary pacer implantation. Initially, she was empirically started on meropenem $2 \mathrm{~g} \mathrm{q} / 8 \mathrm{~h}$ and daptomycin $8 \mathrm{mg} / \mathrm{kg} / 24 \mathrm{~h}$ to cover nosocomial flora 
in a patient recently discharged. Four days after device removal, 16S rRNA gene PCR sequencing detected M. wolinskyi in all the surgical samples of the device and in the fluid aspirated from the surgical site. Three days later, the microbiologic cultures of wounds and devices were positive for acid-fast bacilli identified as M. wolinskyi using MALDT-TOF and 16S rRNA gene sequencing. Susceptibility tests revealed sensitivity to doxycycline, minocycline, tigecycline, quinolones and amikacin and resistance to cephalosporins, imipenem and tobramycin. The antimicrobial regimen was then changed to oral moxifloxacin $400 \mathrm{mg} / \mathrm{q} 24 \mathrm{~h}$ and doxycycline $100 \mathrm{mg} / \mathrm{q} 12 \mathrm{~h}$ planning, for a total of 6 weeks of treatment. The patient had complete recovery at the end of treatment at home and was also evaluated by the electrophysiology team, which considered a new device was not necessary. She presented no complications or relapse at 1-year follow-up.

\section{DISCUSSION AND REVIEW}

Data on M. wolinskyi infection are limited to only case reports. Generally, it is associated with skin and soft tissue infections, bone infection and bacteremia. The ability of these organisms to cause cardiovascular infections has not, to date, been widely appreciated. However, of 99 articles related to M. wolinskyi, 21 cases of $M$. wolinskyi infection have been identified [6-12] in the review of literature in MEDLINE (source PubMed) from 1999 to April 2020 [7-12], and, including ours but excluding duplicated cases, 11 of them $(52 \%)$ were cardiovascular infections (Supplementary Fig. 1), the characteristics of which are summarized in Table 1 . Male gender was more prevalent $(72.7 \%)$; median age was 55 years. Predominantly, there were surgical wound infections (54.5\%) and sternal osteomyelitis $(33.3 \%)$, followed by infective endocarditis $(27.3 \%)$ and CIED infection (18.2\%). The antibiotic treatment was specified in $81.8 \%$ of cases. Combined therapy was selected in all cases and included a combination of quinolones plus tetracyclines ( \pm aminoglycosides) in $7 / 11$ (64\%), quinolones plus macrolides plus linezolid 1/11 (9\%) and quinolones plus carbapenems plus cotrimoxazole 1/11
(9\%). All cases required surgery. Although only $45.5 \%$ of the studies provided follow-up data, no patients died [9-12].

However, the role of other NTM species in cardiovascular system infections has been emphasized recently. An example of this was the prolonged outbreak of M. chimaera following open heart surgery, which was linked to airborne transmission from contaminated water heater and cooler water tanks and was extensively reported [3-5]. Thereafter, a global awareness-raising campaign, rapid interventions for infection prevention and control programs to reduce the impact of $M$. chimaera in cardiothoracic infections were introduced. Infective endocarditis cases due to Mycobacterium chelonae after prosthetic valve surgery have been also described [6].

The increasing number of reported cases of M. wolinskyi infection can probably be explained by the recent addition to the $16 \mathrm{~S}$ rRNA gene sequencing library rather than by an actual rise in clinical occurrence [2]. However, given the characteristics of $M$. wolinskyi as an environmental Mycobacterium and this higher prevalence in cardiothoracic surgery patients, heart-lung machines might be suspected as a potential source. In fact, a cluster of M. wolinskyi cardiothoracic surgical site infections was reported in 2014 in an academic tertiary-care reference medical center [11]. In their analysis, Nagpal et al. showed an epidemic behavior of M. wolinskyi and described six definite cases of M. wolinskyi wound site infection following cardiothoracic surgery during the outbreak period between 2008 and 2011. They designed a case-control study comparing the 6 cases with 18 controls and performed environmental microbiologic sampling and high-volume water sampling. No differences were observed between groups except cardiac surgery in a specific operating room, which was associated with infection $(p=0.0027)$. They could not establish a definite source in all cases but achieved a reduction of the infection rate when all the potential sources were removed $[11,12]$.

Although it is unclear how the inoculation of M. wolinskyi may occur, the clinical presentation as sternal wound infections, osteomyelitis, mediastinitis, infective endocarditis, vascular 


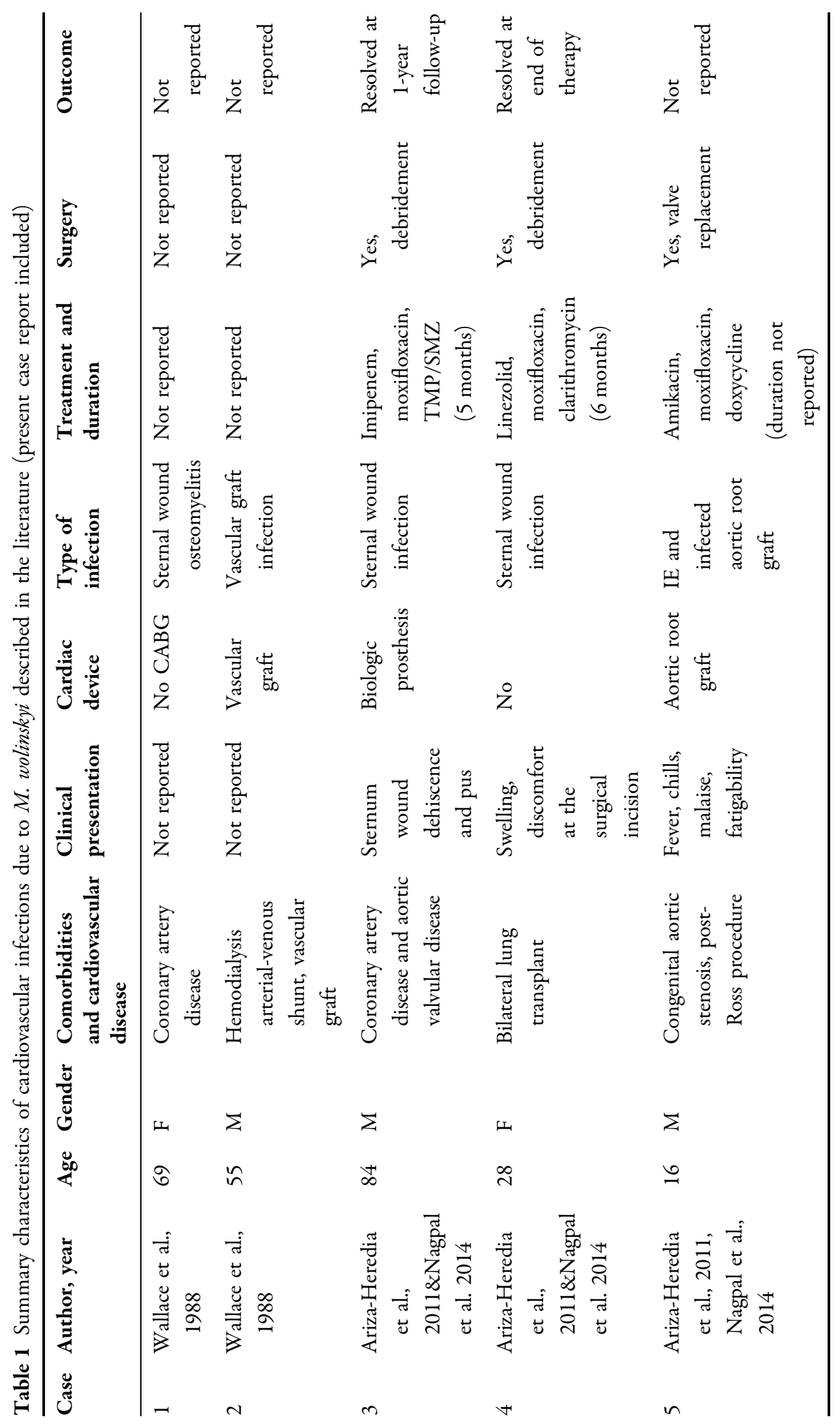




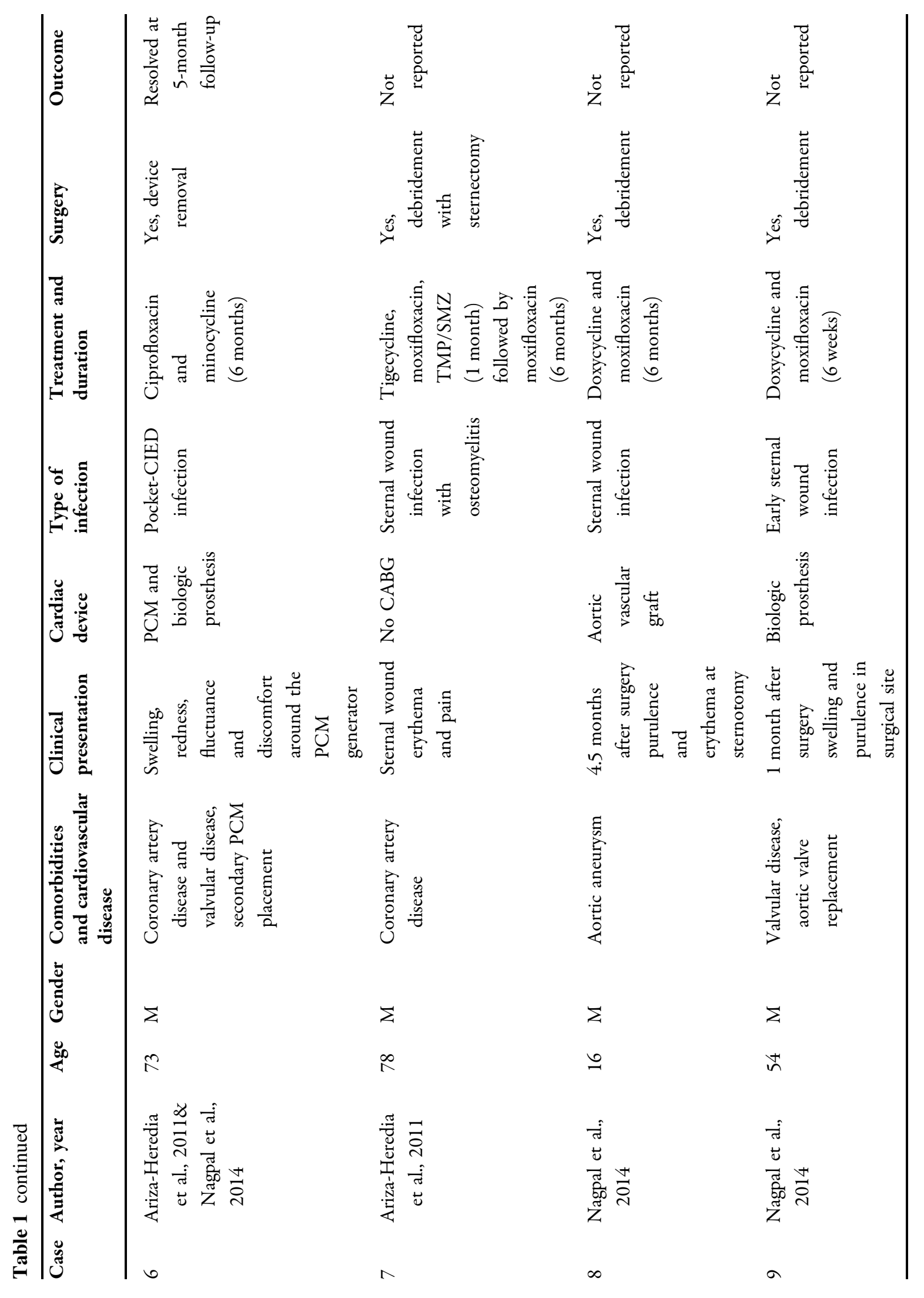




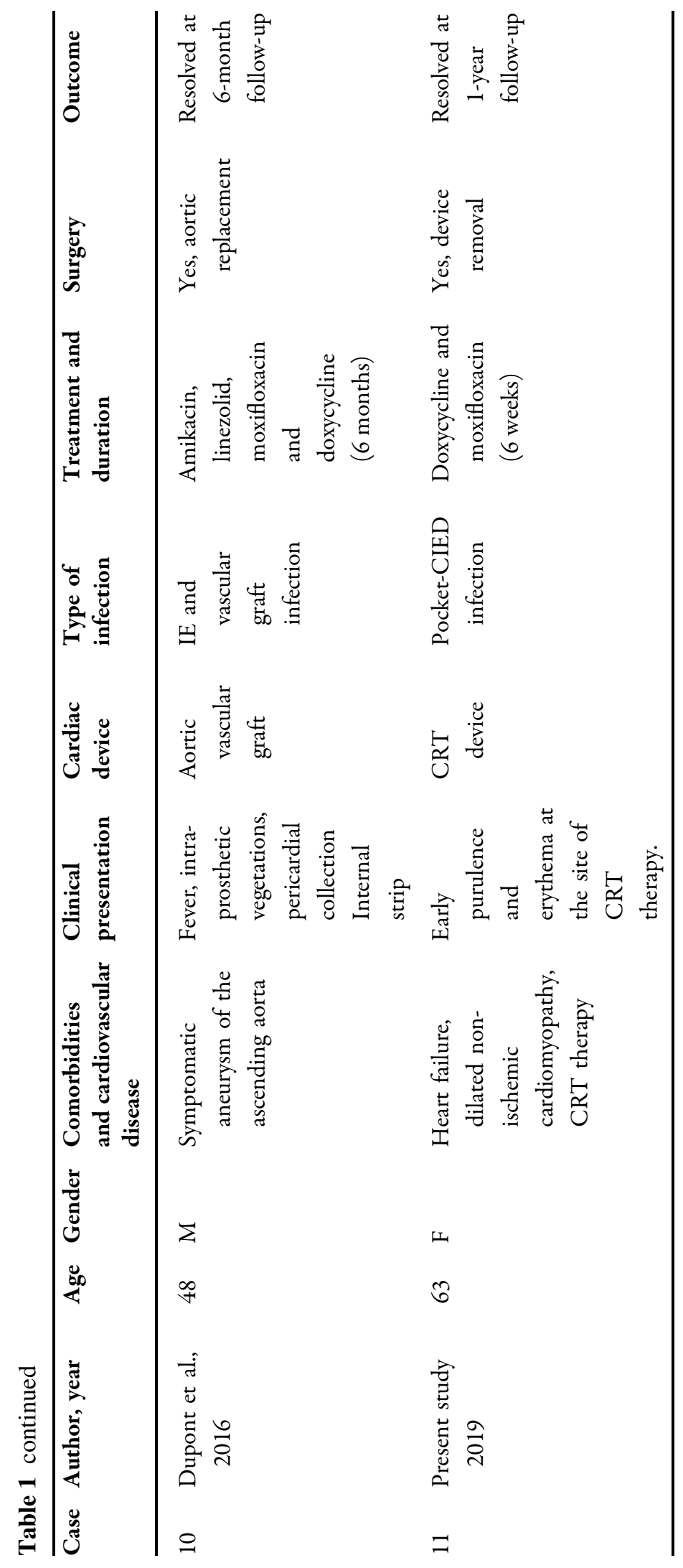


graft infections and CIED infections may suggest that M. wolinskyi could be acquired at the time of surgery rather than in a subsequent exposure to an environmental contaminant. In our case, the potential for contamination in the context of the operating room could not be established. There were several microbiologic samples of the surgical environment from the days preceding and following the patient's procedure, all of which tested negative for NTM.

Finally, the therapeutic strategies and regimen duration to treat these infections are not well established. The typical profile of the in vitro susceptibility of $M$. wolinskyi is susceptibility to amikacin and resistance to tobramycin, which differentiate $M$. wolinskyi from the other members of the Mycobacterium smegmatis group. M. wolinskyi also frequently has intermediate susceptibility to doxycycline and quinolones. Its susceptibility to imipenem, cefoxitin, clarithromycin, linezolid and TMP/ SMZ has been described in recent case reports [13]. As in our case, a preference for the combination of quinolones and doxycycline might be recommended as initial therapy while awaiting susceptibility tests.

\section{CONCLUSION}

In conclusion, NTM cardiovascular infections remain rare, but are increasingly reported. Although available information is still limited, M. wolinskyi seems to be uncommon but might be emerging as it has increasingly been described as a cause of cardiovascular infections, especially in cardiothoracic surgical wounds and CIED infections, directing suspicion toward contamination in the operating room. As more evidence becomes available, it should be observed in the future to conclude whether it is an emergent Mycobacterium in such infections. This must therefore be considered in cases where there is a presumption of cardiovascular infection and an unknown etiology with negative culture for conventional organisms. Likewise, molecular techniques may increase and accelerate diagnoses.

\section{ACKNOWLEDGEMENTS}

We express our appreciation to all members of the Cardiovascular Infectious Diseases team and Microbiology Department for their contributions to this study and their precious work and efforts in clinical care and daily practice with all our patients.

Members of the Hospital Clinic Endocarditis Study Group, Barcelona, Spain, are José M. Miró, Juan Ambrosioni, Marta Hernández-Meneses, Asunción Moreno (Infectious Diseases Service); Cristina Garcia de la Mària, María Alexandra Cañas, Javier Garcia (Experimental Endocarditis Laboratory); Manel Almela, Jordi Vila (Microbiology Service); Eduard Quintana, Elena Sandoval, Carlos Falces, Barbara Vidal, José M. Tolosana, Daniel Pereda, Jose Ortiz, Rut Andrea, Manuel Castella, Marta Sitges (Cardiovascular Institute); Irene Rovira (Anesthesiology Department); David Fuster, Andrés Perisinotti (Nuclear Medicine Service); Jose Ramírez (Pathology Department); Merce Brunet (Toxicology Service); Dolors Soy (Pharmacy Service); Pedro Castro, Adrián Téllez (Intensive Care Unit); and Jaime Llopis (Department of Statistics, Faculty of Biology, University of Barcelona).

Funding. This work was supported by the Ministerio de Sanidad y Consumo of Spain (FIS NCT00871104, Instituto de Salud Carlos III). Institut d'Investigacions Biomèdiques Pi i Sunyer (IDIBAPS) Barcelona (Spain) provided a personal intensification research grant \#INT15/ 00168 and a personal 80:20 research grant to JMM during 2017-2021. MHM received a Rio Hortega Research Grant (CM17/00062) from the "Instituto de Salud Carlos III" and the "Ministerio de Economia y Competitividad," Madrid, Spain, in 2018-2020. No Rapid Service Fee was received by the journal for the publication of this article.

Authorship. All the authors contributed to conception and design, acquisition of data, drafting of the article, critical revision, and final approval of the manuscript. 
Disclosures. Jose M. Miró has received consulting honoraria and/or research grants from AbbVie, Bristol-Myers Squibb, Cubist, Novartis, Gilead Sciences and ViiV all outside the current work. Juan Ambrosioni has received consulting honoraria and/or research grants from Gilead Sciences, Janssen Pharmaceuticals and ViiV, all outside the current work. Marta HernándezMeneses, Julian González-Martin, Daiana Agüero, Jose M. Tolosana, Elena Sandoval, Carles Falces, Rodolfo San Antonio, Bárbara Vidal and Asunción Moreno have nothing to disclose.

Compliance with Ethics Guidelines. The patient has provided informed consent for the publication of this case report.

Open Access. This article is licensed under a Creative Commons Attribution-NonCommercial 4.0 International License, which permits any non-commercial use, sharing, adaptation, distribution and reproduction in any medium or format, as long as you give appropriate credit to the original author(s) and the source, provide a link to the Creative Commons licence, and indicate if changes were made. The images or other third party material in this article are included in the article's Creative Commons licence, unless indicated otherwise in a credit line to the material. If material is not included in the article's Creative Commons licence and your intended use is not permitted by statutory regulation or exceeds the permitted use, you will need to obtain permission directly from the copyright holder. To view a copy of this licence, visit http://creativecommons.org/licenses/by$\mathrm{nc} / 4.0 /$.

\section{REFERENCES}

1. Brown BA, Springer B, Steingrube VA, et al. Mycobacterium wolinskyi sp. nov. and Mycobacterium goodii sp. nov., two new rapidly growing species related to Mycobacterium smegmatis and associated with human wound infections: a cooperative study from the International Working Group on Mycobacterial Taxonomy. Int J Syst Bacteriol. 1999;49:1493-1511.
2. Crago B, Ferrato C, Drews SJ, et al. Surveillance and molecular characterization of non-tuberculous mycobacteria in a hospital water distribution system over a three-year period. J Hosp Infect. 2014;8: $59 \mathrm{e} 62$.

3. Kohler P, Kuster SP, Bloemberg G, et al. Healthcareassociated prosthetic heart valve, aortic vascular graft, and disseminated Mycobacterium chimaera infections subsequent to open heart surgery. Eur Heart J. 2015;36(40):2745-53.

4. Williamson D, Howden B, Stiner T. Mycobacterium chimaera spread from heating and cooling units in heart surgery. N Engl J Med. 2017;376:600-6002.

5. Van Ingen J, Kohl TA, Kranzer K, et al. Global outbreak of sever Mycobacterium chimaera disease after cardiac surgery: a molecular epidemiological study. Lancet Infect Dis. 2017;17(10):1033-41.

6. Wallace RJ Jr, Nash DR, Tsukamura M, et al. Human disease due to Mycobacterium smegmatis. J Infect Dis. 1988;158:52-9.

7. Bhatnagar N, Poojary A, Manlar A, et al. Mycobacterium wolinskyi: a rare strain isolated in a persistent prosthetic knee joint infection: a case report. JBJS Case Connect. 2019. https://doi.org/10.2106/JBJS. CC.18.00315.

8. Fujikura H, Kasahara K, Ogawa Y, et al. Mycobacterium wolinskyi peritonitis after peritoneal catheter embadment surgery. Intern Med. 2017;56(22): 3097-101.

9. Bossart S, Schell B, Kerl K, et al. Ulcers as a sign of skin infection with Mycobacterium wolinskyi: report of a case and review of the literature. Case Rep Dermatol. 2016;8:151-5.

10. Ariza-Heredia Ella J, Dababneh Ala S, Wilhelm Mark $\mathrm{P}$, et al. Mycobacterium wolinskyi: a case series and review of the literature. Diagn Microbiol Infect Dis. 2011;71:421-7.

11. Avish Nagpal, Jean E. Wentink, Elie F. Berbari, et al. A cluster of Mycobacterium wolinskyi surgical site infections at an academic medical center. Infect Control Hosp Epidemiol 35(9(September 2014)): 1169-1175.

12. Dupont C, Terrub D, Aguilhon S, et al. Source-case investigation of Mycobacterium wolinskyi cardiac surgical site infection. J Hosp Infect. 2016;93: 235-239.

13. Chen YC, Jou R, Huang WL, et al. Bacteremia caused by Mycobacterium wolinskyi. Emerg Infect Dis. 2008;14:1818-9. 